\title{
Quantum Teleportation with a Complete Bell State Measurement
}

\author{
Yoon-Ho Kim, ${ }^{*}$ Sergei P. Kulik, ${ }^{\dagger}$ and Yanhua Shih \\ Department of Physics, University of Maryland, Baltimore County, Baltimore, Maryland 21250
}

(Submitted to PRL)

We report a quantum teleportation experiment in which nonlinear interactions are used for the Bell state measurements. The experimental results demonstrate the working principle of irreversibly teleporting an unknown arbitrary quantum state from one system to another distant system by disassembling into and then later reconstructing from purely classical information and nonclassical EPR correlations. The distinct feature of this experiment is that all four Bell states can be distinguished in the Bell state measurement. Teleportation of a quantum state can thus occur with certainty in principle.

PACS Number: 03.65.Bz, 03.67.Hk, 42.50.Dv, 42.65.Ky

The idea of quantum teleportation is to utilize the nonlocal correlations between an Einstein-Podolsky-Rosen pair of particles [1] to prepare a quantum system in some state, which is the exact replica of an arbitrary unknown state of a distant individual system [2]. Three experiments in this direction were published recently 33 - 5].

The following conditions must be satisfied in any claim for quantum teleportation: (i) the input quantum state, which is teleported in the experiment must be an arbitrary state, (ii) there must be an output quantum state which is an "instantaneous copy" of the input quantum state, and (iii) the Bell state measurement (BSM) must be able to distinguish the complete set of the orthogonal Bell states so that the input state can be teleported with certainty.

In this Letter, we experimentally demonstrate a quantum teleportation scheme which satisfies all three of the above conditions. The input state is an arbitrary polarization state and the BSM can distinguish all four orthogonal Bell states so that the state has a $100 \%$ certainty to be teleported in principle. This is because the BSM is based on nonlinear interactions which are necessary and non-trivial physical processes for correlating the input state and the entangled EPR pair 6. 7].

The basic elements of the experiment are schematically shown in Fig. 1. Just as the original proposal of quantum teleportation [2], it consists of four essential parts: (a) the input state, (b) the EPR pair, (c) Alice (who performs the BSM of the input state and her EPR particle), and (d) Bob (who carries out unitary operations on his EPR particle). The input quantum state is an arbitrary polarization state given by,

$$
\left|\Psi_{1}\right\rangle=\alpha\left|0_{1}\right\rangle+\beta\left|1_{1}\right\rangle
$$

where $|0\rangle$ and $|1\rangle$ represent the two orthogonal linear polarization bases (specifically in this paper) $|H\rangle$ (horizontal) and $|V\rangle$ (vertical) respectively. $\alpha$ and $\beta$ are two arbitrary complex amplitudes with respect to the $|0\rangle$ and

\footnotetext{
${ }^{*}$ Email: yokim@umbc.edu

${ }^{\dagger}$ Permanent Address: Department of Physics, Moscow State University, Moscow, Russia
}

$|1\rangle$ bases and they satisfy the condition $|\alpha|^{2}+|\beta|^{2}=1$. The EPR pair shared by Alice and Bob is prepared by spontaneous parametric down conversion (SPDC) as,

$$
\left|\Psi_{23}\right\rangle=\frac{1}{\sqrt{2}}\left\{\left|0_{2} 0_{3}\right\rangle-\left|1_{2} 1_{3}\right\rangle\right\}
$$

with the subscripts 2 and 3 as labeled in Fig. [1 8]. The complete state of the three particles before Alice's measurement is then,

$$
\begin{aligned}
\left|\Psi_{123}\right\rangle & =\frac{\alpha}{\sqrt{2}}\left\{\left|0_{1} 0_{2} 0_{3}\right\rangle-\left|0_{1} 1_{2} 1_{3}\right\rangle\right\} \\
& +\frac{\beta}{\sqrt{2}}\left\{\left|1_{1} 0_{2} 0_{3}\right\rangle-\left|1_{1} 1_{2} 1_{3}\right\rangle\right\} .
\end{aligned}
$$

The four Bell states which form a complete orthonormal basis for both particle 1 and particle 2 are usually represented as,

$$
\begin{aligned}
& \left|\Phi_{12}^{( \pm)}\right\rangle=\frac{1}{\sqrt{2}}\left\{\left|0_{1} 0_{2}\right\rangle \pm\left|1_{1} 1_{2}\right\rangle\right\} \\
& \left|\Psi_{12}^{( \pm)}\right\rangle=\frac{1}{\sqrt{2}}\left\{\left|0_{1} 1_{2}\right\rangle \pm\left|1_{1} 0_{2}\right\rangle\right\} .
\end{aligned}
$$

State (3) can now be re-written in the following form based on the above orthonormal Bell states,

$$
\begin{aligned}
& \left|\Psi_{123}\right\rangle=\frac{1}{2}\left\{\quad\left|\Phi_{12}^{(+)}\right\rangle\left(\alpha\left|0_{3}\right\rangle-\beta\left|1_{3}\right\rangle\right)\right. \\
& +\left|\Phi_{12}^{(-)}\right\rangle\left(\alpha\left|0_{3}\right\rangle+\beta\left|1_{3}\right\rangle\right) \\
& +\left|\Psi_{12}^{(+)}\right\rangle\left(-\alpha\left|1_{3}\right\rangle+\beta\left|0_{3}\right\rangle\right) \\
& \left.+\left|\Psi_{12}^{(-)}\right\rangle\left(-\alpha\left|1_{3}\right\rangle-\beta\left|0_{3}\right\rangle\right) \quad\right\} .
\end{aligned}
$$

To teleport the state of particle 1 to particle 3 reliably, Alice must be able to distinguish her four Bell states by means of the BSM performed on particle 1 and her EPR particle (particle 2). She then tells Bob through a classical channel to perform a corresponding linear unitary operation on his EPR particle (particle 3) to obtain an exact replica of the state of particle 1 . This completes the process of quantum teleportation.

The distinct feature of the scheme shown in Fig.11 is that the BSM is based on nonlinear interactions: optical Sum Frequency Generation (SFG) (or "upconversion"). Four SFG nonlinear crystals are used for "measuring" 
and "distinguishing" the complete set of the four Bell states. Photon 1 and photon 2 may interact either in the two type-I crystals or in the two type-II crystals to generate a higher frequency photon (labeled as photon 4). The projection measurements on photon 4 (either at the $45^{\circ}$ or at the $135^{\circ}$ direction) correspond to the four Bell states of photon 1 and photon $2,\left|\Phi_{12}^{( \pm)}\right\rangle$and $\left|\Psi_{12}^{( \pm)}\right\rangle$.

Let us now discuss the BSM in detail (see Fig.11). The first type-I SFG crystal converts two $|V\rangle$ polarized photons $\left|1_{1} 1_{2}\right\rangle$ into a single horizontal polarized photon $\left|H_{4}\right\rangle$. Likewise, the second type-I SFG crystal converts two $|H\rangle$ polarized photons $\left|0_{1} 0_{2}\right\rangle$ into a single vertical polarized photon $\left|V_{4}\right\rangle$. The first and the last terms on the righthand side in Eq.(3) thus become,

$$
\left|\Psi_{43}\right\rangle=\alpha\left|V_{4} 0_{3}\right\rangle-\beta\left|H_{4} 1_{3}\right\rangle \text {. }
$$

Dichroic beamsplitter $M$ reflects only SFG photons to the $45^{\circ}$ polarization projector $G_{1}$. Two detectors $D_{4}^{I}$ and $D_{4}^{I I}$ are placed at the $45^{\circ}$ and $135^{\circ}$ output ports of $G_{1}$ respectively. Denoting the $45^{\circ}$ and $135^{\circ}$ polarization bases by $\left|45^{\circ}\right\rangle$ and $\left|135^{\circ}\right\rangle$, the state $\left|\Psi_{43}\right\rangle$ may be rewritten as,

$$
\begin{aligned}
\left|\Psi_{43}\right\rangle= & \frac{1}{\sqrt{2}}\left\{\left|45^{\circ}\right\rangle_{4}\left(\alpha\left|0_{3}\right\rangle-\beta\left|1_{3}\right\rangle\right)\right. \\
& \left.+\left|135^{\circ}\right\rangle_{4}\left(\alpha\left|0_{3}\right\rangle+\beta\left|1_{3}\right\rangle\right)\right\}
\end{aligned}
$$

which gives,

$$
\begin{gathered}
|\Psi\rangle_{3 \mid D_{4}^{I}}=\alpha\left|0_{3}\right\rangle-\beta\left|1_{3}\right\rangle \\
|\Psi\rangle_{3 \mid D_{4}^{I I}}=\alpha\left|0_{3}\right\rangle+\beta\left|1_{3}\right\rangle
\end{gathered}
$$

i.e., if detector $D_{4}^{I}\left(45^{\circ}\right)$ is triggered, the quantum state of Bob's EPR photon (photon 3) is:

$$
\left|\Psi_{3}\right\rangle=\alpha\left|0_{3}\right\rangle-\beta\left|1_{3}\right\rangle
$$

and, if detector $D_{4}^{I I}\left(135^{\circ}\right)$ is triggered, the quantum state of Bob's photon is:

$$
\left|\Psi_{3}\right\rangle=\alpha\left|0_{3}\right\rangle+\beta\left|1_{3}\right\rangle
$$

As we have analyzed above, the $45^{\circ}$ and the $135^{\circ}$ polarized type-I SFG components in Eq. (5) correspond to the superposition of $\left|0_{1} 0_{2}\right\rangle$ and $\left|1_{1} 1_{2}\right\rangle$ which are the respective Bell states $\left|\Phi_{12}^{(+)}\right\rangle$and $\left|\Phi_{12}^{(-)}\right\rangle$.

Similarly, the other two Bell states are distinguished by the type-II SFG's. The states $\left|0_{1} 1_{2}\right\rangle$ and $\left|1_{1} 0_{2}\right\rangle$ are made to interact in the first and the second type-II SFG crystals respectively to generate a higher frequency photon with either horizontal (the first type-II SFG) or vertical (the second type-II SFG) polarization. A $45^{\circ}$ polarization projector $G_{2}$ is used after the type-II SFG crystals and two detectors $D_{4}^{I I I}$ and $D_{4}^{I V}$ are placed at the $45^{\circ}$ and the $135^{\circ}$ output ports of $G_{2}$ respectively. On the new bases of $45^{\circ}$ and $135^{\circ}$ for the SFG photon, the second and the third terms on the right-hand side in Eq.(3) thus become,

$$
\begin{aligned}
\left|\Psi_{43}\right\rangle= & \frac{1}{\sqrt{2}}\left\{\left|45^{\circ}\right\rangle_{4}\left(-\alpha\left|1_{3}\right\rangle+\beta\left|0_{3}\right\rangle\right)\right. \\
& \left.+\left|135^{\circ}\right\rangle_{4}\left(-\alpha\left|1_{3}\right\rangle-\beta\left|0_{3}\right\rangle\right)\right\},
\end{aligned}
$$

which gives,

$$
\begin{gathered}
|\Psi\rangle_{3 \mid D_{4}^{I I I}}=-\alpha\left|1_{3}\right\rangle+\beta\left|0_{3}\right\rangle, \\
|\Psi\rangle_{3 \mid D_{4}^{I V}}=-\alpha\left|1_{3}\right\rangle-\beta\left|0_{3}\right\rangle,
\end{gathered}
$$

i.e., if detector $D_{4}^{I I I}\left(45^{\circ}\right)$ is triggered, the quantum state of Bob's photon is:

$$
\left|\Psi_{3}\right\rangle=-\alpha\left|1_{3}\right\rangle+\beta\left|0_{3}\right\rangle
$$

and if detector $D_{4}^{I V}\left(135^{\circ}\right)$ is triggered, the quantum state of Bob's photon is:

$$
\left|\Psi_{3}\right\rangle=-\alpha\left|1_{3}\right\rangle-\beta\left|0_{3}\right\rangle
$$

The $45^{\circ}$ and the $135^{\circ}$ polarized type-II SFG components correspond to the superposition of $\left|0_{1} 1_{2}\right\rangle$ and $\left|1_{1} 0_{2}\right\rangle$ which are the Bell states $\left|\Psi_{12}^{(+)}\right\rangle$and $\left|\Psi_{12}^{(-)}\right\rangle$respectively.

To obtain the exact replica of the state of Eq.(11), Bob needs simply to perform a corresponding unitary transformation after learning from Alice which of her four detectors, $D_{4}^{I}, D_{4}^{I I}, D_{4}^{I I I}$, or $D_{4}^{I V}$, has triggered [9].

To demonstrate the working principle of this scheme, we measure the joint detection rates between detectors $D_{4}^{I}-D_{3}, D_{4}^{I I}-D_{3}, D_{4}^{I I I}-D_{3}$ and $D_{4}^{I V}-D_{3}$, where $D_{3}$ is Bob's detector (see Fig.4). In these measurements we choose the input state $\left|\Psi_{1}\right\rangle$ as a linear polarization state. For a fixed input polarization state, the angle of the polarization analyzer $A_{3}$ which is placed in front of Bob's detector is rotated and the joint detection rates are recorded. Figure 2 shows two typical data sets for $D_{4}^{I}-D_{3}$ and $D_{4}^{I I}-D_{3}$. The input polarization state is $45^{\circ}$. Clearly, these data curves confirm Eq.(6). The different phases of the two curves reflect the phase difference between the two states in Eq.(6). Experimental data for $D_{4}^{I I I}-D_{3}$ and $D_{4}^{I V}-D_{3}$ show similar behavior, see Fig. 3, which confirm Eq.(8).

We now discuss the details of the experimental setup. The schematic of the experimental setup is shown in Fig. 4. The input polarization state is prepared by using a $\lambda / 2$ plate from a femtosecond laser pulse (pulse width $\approx 100 \mathrm{fsec}$ and central wavelength $=800 \mathrm{~nm}$ ) [10. The EPR pair (730nm-885nm photon pair) is generated by two non-degenerate type-I SPDC's. The optical axes of the first and the second SPDC crystals are oriented in the respective horizontal $(\odot)$ and vertical $(\uparrow)$ directions. The SPDC crystals are pumped by a $45^{\circ}$ polarized $100 \mathrm{fsec}$ laser pulse with $400 \mathrm{~nm}$ central wavelength. The BBO crystals (each with thickness $3.4 \mathrm{~mm}$ ) are cut for collinear non-degenerate phase matching. Since the two crystals 
are pumped equally, the SPDC pair can be generated either in the first BBO as $\left|V_{885}\right\rangle_{2}\left|V_{730}\right\rangle_{3}\left(\left|1_{2} 1_{3}\right\rangle\right)$ or in the second BBO as $\left|H_{885}\right\rangle_{2}\left|H_{730}\right\rangle_{3}\left(\left|0_{2} 0_{3}\right\rangle\right)$ with equal probability ( 885 and 730 refer to the wavelengths in nanometer). In order to prepare an EPR state in the form of Eq.(2) (a Bell state), these two amplitudes have to be quantum mechanically "indistinguishable" and have the expected relative phase. A Compensator $(\mathrm{C}-1)$ is used for this purpose and it consists of two parts: a thick quartz rod and two thin plates. The thick quartz rod is used to compensate the time delay between the two amplitudes $\left|1_{2} 1_{3}\right\rangle$ and the $\left|0_{2} 0_{3}\right\rangle$, and two thin quartz plates are used to adjust the relative phase between them by angular tilting. A dichroic beamsplitter $D B S$ is placed behind the SPDC crystals to separate and send the photon $2(885 \mathrm{~nm})$ and photon $3(730 \mathrm{~nm})$ to Alice and Bob respectively. To check the EPR state, a flipper mirror $F M$ is used to send the photon $2(885 \mathrm{~nm})$ to a photoncounting detector $D_{2}$ for EPR correlation measurement. Both the space-time and polarization correlations must be checked before teleportation measurements, in order to be certain of having high degree EPR entanglement and the expected relative phase between the $\left|1_{2} 1_{3}\right\rangle$ and the $\left|0_{2} 0_{3}\right\rangle$ amplitudes (see Ref. [11] for details). Once the EPR state in Eq.(2) is prepared, $F M$ is flipped-down and photon $2(885 \mathrm{~nm})$ is given to Alice for BSM with photon 1.

The BSM consists of four SFG nonlinear crystals, two $45^{\circ}$ projectors $\left(G_{1}\right.$ and $\left.G_{2}\right)$, four single photon counting detectors $\left(D_{4}^{I}, D_{4}^{I I}, D_{4}^{I I I}\right.$, and $\left.D_{4}^{I V}\right)$ and two compensators as well as other necessary optical components. The input photon $(800 \mathrm{~nm})$ and photon $2(885 \mathrm{~nm})$ may either interact in the two type-I or in the two type-II SFG crystals. Two pairs of lenses $(L)$ are used as telescopes to focus the input beams onto the crystals. The vertical (horizontal) polarized amplitudes of the input photon $(800 \mathrm{~nm})$ and the vertical (horizontal) polarized photon $2(885 \mathrm{~nm})$ interact in the first (second) type-I SFG to generate a $420 \mathrm{~nm}$ horizontal (vertical) polarized photon 12]. The horizontal (vertical) polarized amplitudes of the input photon and the vertical (horizontal) polarized photon 2 interact in the first (second) type-II SFG to generate a $420 \mathrm{~nm}$ horizontal (vertical) polarized photon. The $420 \mathrm{~nm}$ photons generated in the type-I SFG process is reflected to detectors $D_{4}^{I}$ and $D_{4}^{I I}$ (after passing through C-2 and a $45^{\circ}$ polarization projector $G_{1}$ ) by a dichroic beamsplitter $D B S_{2}$ and similarly for the $420 \mathrm{~nm}$ photons created in two type-II SFG processes. It is very important to design and adjust the Compensators (C-2 and C-3) correctly in order to make the horizontal and the vertical components of the $420 \mathrm{~nm}$ SFG quantum mechanically indistinguishable and to attain the expected relative phase. These two compensators are similar to C-1.

Since the input state (photon 1) and photon 2 should overlap inside the SFG crystals exactly, a prism is used to adjust the path-length of the input pulse [13]. $M_{1}$ is a dichroic mirror which reflects the $800 \mathrm{~nm}$ photons while transmitting the $885 \mathrm{~nm}$ ones. In order to be sure that the SFG process occurs with a single-photon input, we measured the coincidence counting rate between one of Alice's detectors and Bob's detector $D_{3}$ by moving the position of the prism. Fig. 同 shows a typical data curve of the measurement. It is clear that SFG only occurs when the input pulse (photon 1) and photon 2 (singlephoton created by the SPDC process) overlap perfectly inside the SFG crystals [14].

Readers might have noticed that the efficiency in the teleportation measurement is a lot lower than the SFG demonstration. The reason why we get such a low coincidence counting rate in Figs.2 and 3 as compared to Fig. 5 is that very small pinholes had to be placed in front of Alice's detectors for the teleportation measurement to ensure good spatial mode overlap. The improvements of the SFG and the collection efficiencies while preserving good spatial mode overlap are now underway.

In summary, we have shown a proof-of-principle experimental demonstration of quantum teleportation with complete a Bell state measurement. The two main features lie at the heart of our scheme: (i) EPR-Bohm type quantum correlation and (ii) the BSM using nonlinear interactions. Single photon SFG is used as the BSM and the working principle is demonstrated by observing correlations between the joint measurement of Alice and Bob. In the current experiment, femtosecond laser pulses are used to prepare the input polarization state to reduce data collection time. Recent research on nonlinear optics at low light levels may enable high-efficiency SFG at single-photon level in the near future [15].

We would like to thank C.H. Bennett and M.H. Rubin for helpful discussions. This work was supported in part by the Office of Naval Research, ARDA, and the National Security Agency.

[1] A. Einstein, B. Podolsky, and N. Rosen, Phys. Rev. 47, 777 (1935).

[2] C.H. Bennett et al., Phys. Rev. Lett. 70, 1895 (1993).

[3] D. Bouwmeester et al., Nature 390, 575 (1997).

[4] D. Boschi et al., Phys. Rev. Lett. 80, 1121 (1998).

[5] A. Furusawa et al., Science 282, 706 (1998).

[6] L. Vaidman and N. Yoran, Phys. Rev. A 59, 116 (1999); N. Lütkenhaus, J. Calsamiglia, and K.-A. Suominen, ibid. 59, 3295 (1999).

[7] D.N. Klyshko, JETP 87, 639 (1998).

[8] Note that any one of the four Bell states can be used for this purpose.

[9] Complete BSM using SFG is also useful for other applications, see C.H. Bennett and S.J. Wiesner, Phys. Rev. Lett. 69, 2881 (1992).

[10] In this experiment, the input state is a polarization state of a femtosecond laser pulse. Only one out of approximately $10^{10}$ photons, all in the same polarization state, in each laser pulse is actually "upconverted" in the SFG process. It can be easily shown that each photon has the polarization state of Eq.(1) by considering the correspondence principle. What is being teleported is the state or 
qubit associated with this photon. (Note, quantum teleportation does not teleport the "quantum" but rather, the state of the quantum). In principle, it does not prevent one to use a single-photon qubit as the input state in this experiment. Due to the low efficiency of SFG, one needs to wait a much longer time for teleportation to occur.

[11] Y.-H. Kim, S.P. Kulik, and Y.H. Shih, Phys. Rev. A 62, $011802(\mathrm{R})$, \protect \vrule width0pt\protect \href\{http://arxiv

[12] Note that there are five different wavelengths which are tunable as long as the two phase matching conditions (for SPDC and for SFG) are satisfied. Also five independent relative phases which affect the output state can be varied.

[13] 800nm input pulse and 400nm pump pulse (which pumps the SPDC crystals) are actually drawn from a single Ti:Sapphire laser to ensure that they have the same repetition period.

[14] The efficiency of SFG (from the SPDC photons) is roughly estimated to be $0.1 \sim 1 \%$. The SFG crystals used for the data shown in this paper are BBO's with $2 \mathrm{~mm}$ thickness.

[15] S.E. Harris and L.V. Hau, Phys. Rev. Lett. 82, 4611 (1999).

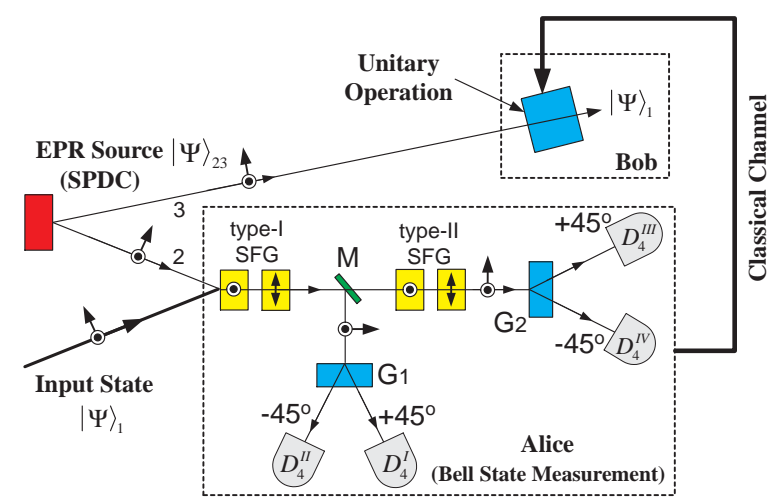

FIG. 1. Principle schematic of quantum teleportation with a complete BSM. Nonlinear interactions (SFG) are used to perform the BSM. $\odot$ and $\uparrow$ represent the respective horizontal and vertical orientations of the optic axes of the crystals.

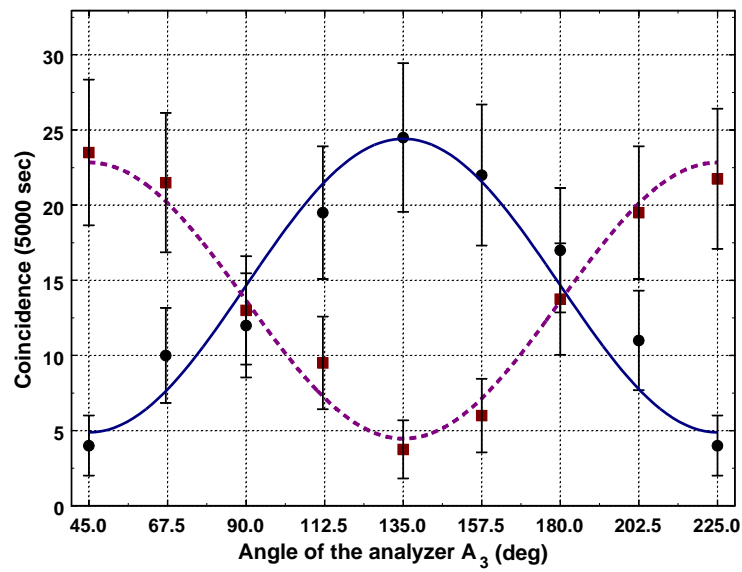

FIG. 2. Solid line (circled data points) is the joint detection rate $D_{4}^{I}-D_{3}$ for $45^{\circ}$ linear polarization as an input state. Dashed line (square data points) is for $D_{4}^{I I}-D_{3}$ for the same input state. The expected $\pi$ phase shift is clearly demonstrated.

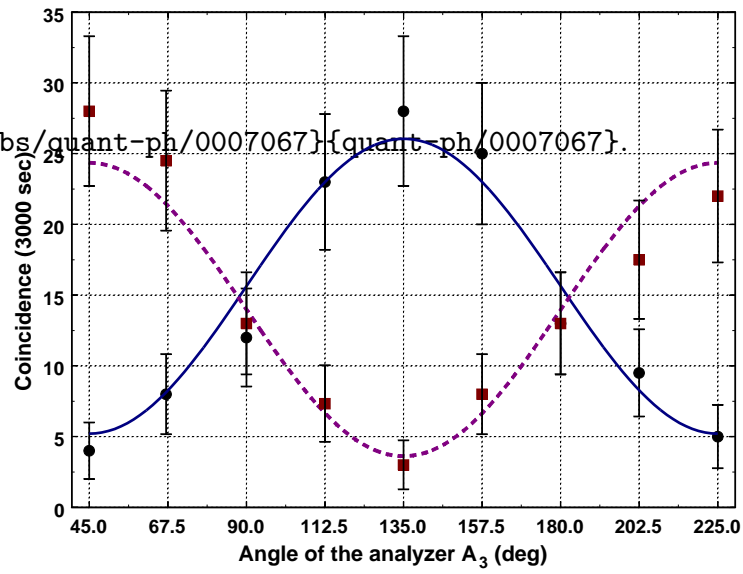

FIG. 3. Solid line (circled data points) is the joint detection rate $D_{4}^{I I I}-D_{3}$ and dashed line (square data points) is for $D_{4}^{I V}-D_{3}$. Again, the expected $\pi$ phase shift is clearly demonstrated.

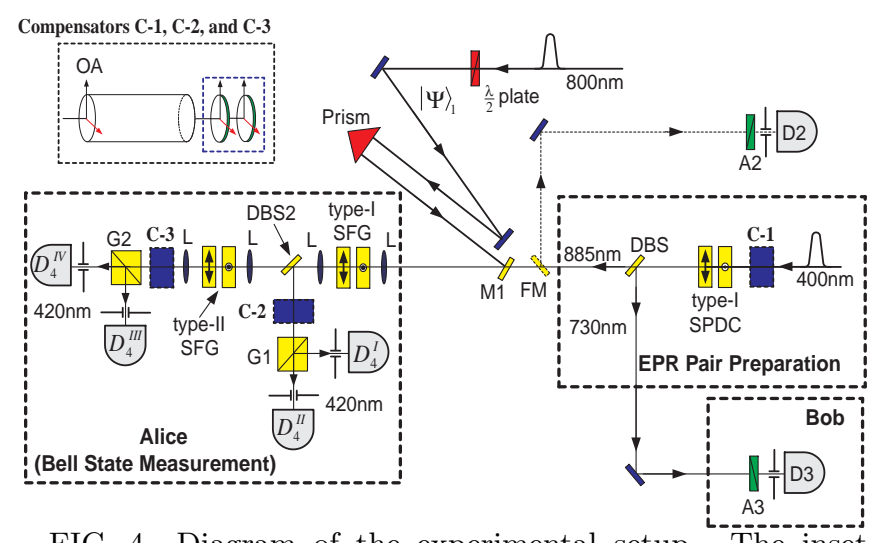

FIG. 4. Diagram of the experimental setup. The inset shows the details of the compensators. See text for details.

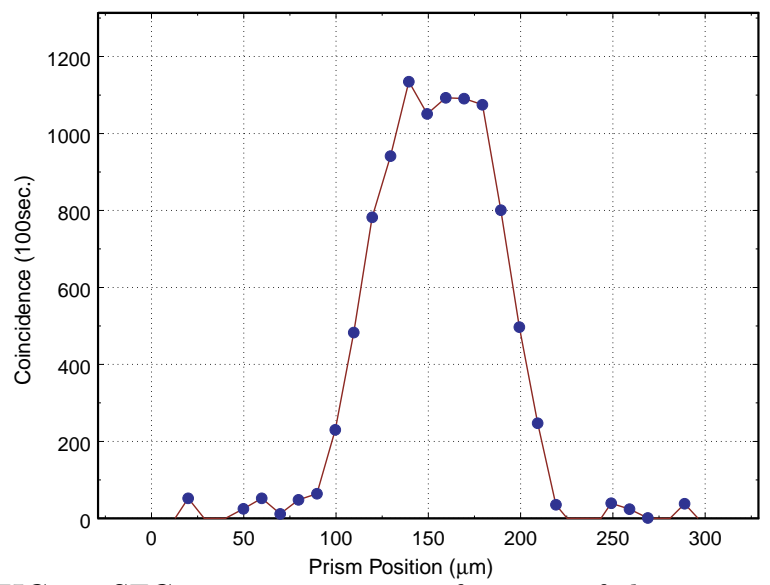

FIG. 5. SFG measurement as a function of the prism position. SFG is observed only when the input pulse and the SPDC photons overlap exactly inside the crystals. 